\title{
Synchronization of Fractional-Order Chaotic Systems with Gaussian Fluctuation by Sliding Mode Control
}

\author{
Yong $\mathrm{Xu}$ and Hua Wang \\ Department of Applied Mathematics, Northwestern Polytechnical University, Xian 710072, China \\ Correspondence should be addressed to Yong Xu; hsux3@nwpu.edu.cn
}

Received 4 September 2013; Accepted 23 October 2013

Academic Editor: Jinhu Lü

Copyright (c) 2013 Y. Xu and H. Wang. This is an open access article distributed under the Creative Commons Attribution License, which permits unrestricted use, distribution, and reproduction in any medium, provided the original work is properly cited.

\begin{abstract}
Chaotic systems are always influenced by some uncertainties and external disturbances. This paper investigates the problem of practical synchronization of fractional-order chaotic systems with Gaussian fluctuation. A fractional integral (FI) sliding surface is proposed for synchronizing the uncertain fractional-order system, and then the sliding mode control technique is carried out to realize the synchronization of the given systems. One theorem about sliding mode controller is presented to prove that the proposed controller can make the system achieve synchronization. As a case study, the presented method is applied to the fractional-order Chen-Lü system, and simulation results show that the proposed control approach is capable to go against Gaussian noise well.
\end{abstract}

\section{Introduction}

Synchronization, which means "things occur at the same time or operate in unison," has received a great deal of interest among scientists from various fields in the last few years, especially in fractional-order chaotic systems [1-4]. It has been recognized that many systems in interdisciplinary fields can be elegantly described by fractional-order differential equations, such as viscoelastic materials [5], electrical circuits [6], population models [7], and financial systems [8]. Meanwhile, most of precious studies have shown that some fractional-order systems exhibit chaotic behavior [914]. In particular, fractional-order chaotic behavior has wide promising applications in information encryption, image processing, secure communication, and so forth [15-18]. Therefore, synchronization of fractional chaotic systems starts to attract increasing attentions because it has a wide range of applications like the traditional (integer order) chaotic synchronization, which has been used in secure communication [19], complex dynamical network [20], and so on

A basic configuration for chaos synchronization is the drive-response pattern, where the response of chaotic system must track the drive chaotic trajectory. Some approaches based on this configuration have been attained to achieve chaos synchronization in fractional-order chaotic systems, such as Pecora and Carroll (PC) control [21], active control [22], adaptive control [23, 24], sliding mode control (SMC) [25], and a scalar transmitted signal method [26], in which the sliding mode controller has some attractive advantages, including: (i) fast dynamic responses and good transient performance; (ii) external disturbance rejection; and (iii) insensitivity to parameter variations and model uncertainties $[27,28]$. In addition, SMC method plays an important role in the application to practical problems. For example, in [29], Tavazoei and Haeri proposed a controller based on active sliding mode theory to synchronize fractional-order chaotic systems in master-slave structure. In [2], the problem of modified projective synchronization of fractional-order chaotic system was considered, and finite-time synchronization of nonautonomous fractional-order uncertain chaotic systems was investigated in [30].

All of the methods mentioned above have been used to synchronize the deterministic fractional-order chaotic systems. However, noise-induced synchronization in chaotic systems is a practical phenomenon due to the fact that noises are ubiquitous in natural and synthetic systems, and up till now, it has been studied by many investigators from different areas [31-34]. What is more is that chaotic systems with fractional-order model influenced by random noise will be 
more challenging and difficult. In this investigation, our aim is to synchronize two fractional-order chaotic systems with uncertain environment. To achieve this goal, we propose fractional integral (FI) sliding mode surface which combines the property of fractional-order equation with sliding mode control method. Theoretical analysis about sliding mode controller is presented via series expansion and properties of Gaussian distribution to prove that the proposed controller can make the system synchronize well. Then a numerical example is given to verify the effectiveness of the mentioned method, and the good agreements are also found between the theoretical and the numerical results.

This paper is organized as follows. In Section 2, fractionalorder chaotic systems with random factors and problem formulation are presented. In Section 3, we investigate the design method of sliding mode controller, and one theorem is obtained to prove the effectiveness of proposed controller. One example is presented to carry out the numerical simulations in Section 4. Finally, conclusions are presented to end this paper.

\section{System Description and Problem Formulation}

Consider the following class of fractional-order chaotic system excited by Gaussian white noise, which is described by

$$
D^{\alpha} \mathbf{x}=\mathbf{A}_{1} \mathbf{x}+\mathbf{f}_{1}(\mathbf{x})+\mathbf{h}(\mathbf{x}, t) \mathbf{W}(t),
$$

where $\mathbf{x}=\left[x_{1}, x_{2}, \ldots, x_{n}\right]^{T} \in R^{n}$ denotes the state vector, $\mathbf{A}_{1} \in R^{n \times n}$ is a constant matrix, $\mathbf{f}_{1}: R^{n} \rightarrow R^{n}$ is nonlinear vector function, $\mathbf{h}$ is noise intensity function which is sufficient smooth and bounded, that is, $|\mathbf{h}(\mathbf{x}, t)| \leq H(H$ is a positive constant), and $\mathbf{W}(t)=\left[W_{1}(t), W_{2}(t), \ldots, W_{n}(t)\right]^{T}$ is $n$-dimensional Brownian motion. Accordingly, $\dot{\mathbf{W}}(t)=$ $\left[\dot{W}_{1}, \dot{W}_{2}, \ldots, \dot{W}_{n}\right]^{T}$ is a $n$-dimensional Gaussian white noise vector, in which every two noises are statistical independent. And $\boldsymbol{\alpha}=\left[\alpha_{1}, \alpha_{2}, \ldots, \alpha_{n}\right]^{T} . D^{\alpha}$ denotes the Caputo derivative, which is defined as

$$
\begin{array}{r}
D^{\alpha} f(t)=\frac{1}{\Gamma(n-\alpha)} \int_{0}^{t} \frac{f^{(n)}(\tau)}{(t-\tau)^{\alpha-n+1}} d \tau, \\
\text { for } n-1<\alpha<n
\end{array}
$$

with $\Gamma(z)=\int_{0}^{\infty} e^{-t} t^{z-1} d t$, the Euler's Gamma function.

Let system (1) be the driving system; then response system with a controller $\mathbf{u}(t)=\left[u_{1}(t), u_{2}(t), \ldots, u_{n}(t)\right]^{T}$ is given by

$$
D^{\alpha} \mathbf{y}=\mathbf{A}_{2} \mathbf{y}+\mathbf{f}_{2}(\mathbf{y})+\mathbf{u}(t),
$$

where $\mathbf{y}=\left[y_{1}, y_{2}, \ldots, y_{n}\right]^{T} \in R^{n}$ is state vector, $\mathbf{A}_{2} \in R^{n \times n}$ is a coefficient matrix, and $\mathbf{f}_{2}: R^{n} \rightarrow R^{n}$ is vector function. Let $\mathbf{e}=\left[e_{1}, e_{2}, \ldots, e_{n}\right]^{T}=\mathbf{y}-\mathbf{x}$ be the error of systems (1) and (3). Then, from system (1) and (3), one has the error dynamics:

$$
\begin{aligned}
D^{\boldsymbol{\alpha}} \mathbf{e} & =\mathbf{A}_{2} \mathbf{y}+\mathbf{f}_{2}(\mathbf{y})+\mathbf{u}(t)-\mathbf{A}_{1} \mathbf{x}-\mathbf{f}_{1}(\mathbf{x})-\mathbf{h}(\mathbf{x}, t) \dot{\mathbf{W}} \\
& =\mathbf{A}_{2} \mathbf{e}+\mathbf{F}(\mathbf{x}, \mathbf{y})-\mathbf{h}(\mathbf{x}, t) \dot{\mathbf{W}}+\mathbf{u}(t),
\end{aligned}
$$

where $F(x, y)=\mathbf{f}_{2}(\mathbf{y})-\mathbf{f}_{1}(\mathbf{x})+\left(\mathbf{A}_{2}-\mathbf{A}_{1}\right) \mathbf{x}$.
Thus, the control problem considered in this study is that, for chaotic driving system (1) and response system (3), they are to be synchronized by designing an appropriate controller $\mathbf{u}(t)$ satisfying

$$
\lim _{t \rightarrow \infty}\|\mathbf{e}\|=\lim _{t \rightarrow \infty}\|\mathbf{y}-\mathbf{x}\|=0
$$

where $\|\cdot\|$ is defined as $\|\mathbf{e}(t)\|=\left(E\left[\mathbf{e}(t)^{T} \mathbf{e}(t)\right]\right)^{1 / 2}$ and $E[\cdot]$ is the expected value function.

\section{Sliding Mode Controller Design and Analysis}

In the following context, we will design sliding mode controller to establish synchronization between driving system (1) and response system (3).

3.1. Sliding Mode Controller Design Process. Now, the control input vector $\mathbf{u}(t)$ is defined to eliminate the nonlinear part of the error dynamics:

$$
\mathbf{u}(t)=\mathbf{H}(t)-\mathbf{F}(\mathbf{x}, \mathbf{y}),
$$

where $\mathbf{H}(t)=\mathbf{K w}(t), \mathbf{K}$ is a constant gain matrix, and $\mathbf{w}(t) \epsilon$ $R^{n}$ is the control input that satisfies

$$
\mathbf{w}(t)= \begin{cases}\mathbf{w}^{+}(t) & \mathbf{S}(\mathbf{e}) \geq 0, \\ \mathbf{w}^{-}(t) & \mathbf{S}(\mathbf{e})<0,\end{cases}
$$

in which $\mathbf{S}=\mathbf{S}(\mathbf{e})$ is a switching surface to prescribe the desired sliding mode dynamics.

So the error system (4) is rewritten as

$$
D^{\alpha} \mathbf{e}=\mathbf{A}_{2} \mathbf{e}+\mathbf{H}(t)-\mathbf{h}(\mathbf{x}, t) \dot{\mathbf{W}} .
$$

Here, a new fractional integral (FI) switching surface is given as follows:

$$
\mathbf{S}=D^{\boldsymbol{\alpha}-1} \mathbf{e}-\int_{0}^{t}\left(\mathbf{K}+\mathbf{A}_{2}\right) \mathbf{e}(\tau) d \tau,
$$

where $\mathbf{S}=\left[s_{1}, s_{2}, \ldots, s_{n}\right]^{T}$ and $\mathbf{K}+\mathbf{A}_{2}$ should be stable; namely, the eigenvalues $\lambda_{i}(i=1,2, \ldots, n)$ of matrix $\mathbf{K}+\mathbf{A}_{2}$ are negative $\left(\lambda_{i}<0\right)$.

As we all know, when the system is controllable in the sliding mode, the switching surface should satisfy the following conditions:

$$
\mathbf{S}(\mathbf{e})=\mathbf{0} \text {, }
$$

together with

$$
\dot{\mathbf{S}}(\mathbf{e})=\mathbf{0}
$$

Substituting (8) and (9) into (10b), one obtains

$$
\begin{aligned}
\dot{\mathbf{S}} & =D^{\alpha} \mathbf{e}-\left(\mathbf{K}+\mathbf{A}_{2}\right) \mathbf{e}(t) \\
& =\mathbf{A}_{2} \mathbf{e}+\mathbf{k} \mathbf{w}(t)-\mathbf{h}(\mathbf{x}, t) \dot{\mathbf{W}}-\left(\mathbf{K}+\mathbf{A}_{2}\right) \mathbf{e}(t) \\
& =\mathbf{K w}(t)-\mathbf{h}(\mathbf{x}, t) \dot{\mathbf{W}}-\mathbf{K e}(t) \\
& =\mathbf{0} .
\end{aligned}
$$


Therefore, the equivalent control law is obtained by

$$
\mathbf{w}_{\mathrm{eq}}(t)=\mathbf{e}(t)+\mathbf{K}^{-1} \mathbf{h}(\mathbf{x}, t) \dot{\mathbf{W}} .
$$

In real-world applications, the Gaussian white noise $\dot{\mathbf{W}}$ is uncertain. Therefore, the equivalent control input is modified to

$$
\mathbf{w}_{\mathrm{eq}}(t)=\mathbf{e}(t) .
$$

To design the sliding mode controller, we consider the constant plus proportional rate reaching law $[35,36]$; that, is

$$
\dot{\mathbf{S}}=-r \mathbf{S}-\rho \operatorname{sgn}(\mathbf{S}),
$$

where $\operatorname{sgn}(\mathbf{S})=\left[\operatorname{sgn}\left(s_{1}\right), \operatorname{sgn}\left(s_{2}\right), \ldots, \operatorname{sgn}\left(s_{n}\right)\right]^{T}, r$ and $\rho$ are all positive numbers, and $\operatorname{sgn}(x)$ represents sign function; that is

$$
\operatorname{sgn}(x)= \begin{cases}1, & x>0, \\ 0, & x=0, \\ -1, & x<0\end{cases}
$$

So, we can get the controller

$$
\mathbf{w}(t)=\mathbf{K}^{-1}(\mathbf{K e}-r \mathbf{S}-\rho \operatorname{sgn}(\mathbf{S})) .
$$

Further, according to the control law and the updated law, the controller is given by

$$
\mathbf{u}(t)=\mathbf{K e}-r \mathbf{S}-\rho \operatorname{sgn}(\mathbf{S})-\mathbf{f}_{2}(\mathbf{y})+\mathbf{f}_{1}(\mathbf{x})-\left(\mathbf{A}_{2}-\mathbf{A}_{1}\right) \mathbf{x} .
$$

And the error system can be rewritten in the following differential form:

$$
D^{\boldsymbol{\alpha}} \mathbf{e}=\left(\mathbf{K}+\mathbf{A}_{2}\right) \mathbf{e}(t)-\mathbf{h}(\mathbf{x}, t) \dot{\mathbf{W}}-r \mathbf{S}-\rho \operatorname{sgn}(\mathbf{S}) .
$$

\subsection{Synchronization Analysis}

Theorem 1. If the controller is selected as (17), with suitably selected $r$ and $\rho$, then the synchronization of fractional-order chaotic systems between driving system (1) and response system (3) can be achieved (i.e., the synchronization error converges to zero in the mean square norm).

Proof. Consider a Lyapunov function constructed by the mean square norm of $\mathbf{S}(t)$ and its differential form [37]:

$$
\begin{gathered}
\mathbf{V}=\frac{1}{2}\|\mathbf{S}(t)\|^{2}=\frac{1}{2} E\left[\mathbf{S}^{2}(t)\right], \\
d \mathbf{V}=\frac{1}{2} E\left[d\left(\mathbf{S}^{2}(t)\right)\right] .
\end{gathered}
$$

According to the definition of derivative, it is found that

$$
\begin{aligned}
d\left(\mathbf{S}^{2}(t)\right) & =(\mathbf{S}(t)+d \mathbf{S}(t))^{2}-\mathbf{S}^{2}(t) \\
& =2 \mathbf{S}(t) d \mathbf{S}(t)+d \mathbf{S}(t) d \mathbf{S}(t),
\end{aligned}
$$

While, from (11) and (16), one gets

$$
d \mathbf{S}(t)=(-r \mathbf{S}-\rho \operatorname{sgn}(\mathbf{S})) d t-\mathbf{h}(\mathbf{x}, t) d \mathbf{W} .
$$

Substituting (21) into (20) results in

$$
\begin{aligned}
d\left(\mathbf{S}^{2}(t)\right)= & 2 \mathbf{S}(t)((-r \mathbf{S}-\rho \operatorname{sgn}(\mathbf{S})) d t-\mathbf{h}(\mathbf{x}, t) d \mathbf{W}) \\
& +\mathbf{h}^{2}(\mathbf{x}, t) d t
\end{aligned}
$$

Taking expectations to (22) and using the properties of Brownian motion, we have

$$
\begin{aligned}
& d \mathbf{V}=E[ \mathbf{S}(t)((-r \mathbf{S}-\rho \operatorname{sgn}(\mathbf{S})) d t-\mathbf{h}(\mathbf{x}, t) d \mathbf{W}) \\
&\left.\quad+\frac{1}{2} \mathbf{h}^{2}(\mathbf{x}, t) d t\right] \\
&=E\left[\mathbf{S}(t)((-r \mathbf{S}-\rho \operatorname{sgn}(\mathbf{S})) d t)+\frac{1}{2} \mathbf{h}^{2}(\mathbf{x}, t) d t\right] .
\end{aligned}
$$

Therefore

$$
\begin{aligned}
\dot{\mathbf{V}} & =E\left[\mathbf{S}(t)(-r \mathbf{S}-\rho \operatorname{sgn}(\mathbf{S}))+\frac{1}{2} \mathbf{h}^{2}(\mathbf{x}, t)\right] \\
& \leq-r E\left[\mathbf{S}^{2}\right]-\rho E[|\mathbf{S}|]+\frac{1}{2} H^{2} .
\end{aligned}
$$

Equation (24) implies that as long as suitable $r$ and $\rho$ which satisfy $(1 / 2) H^{2} \leq r E\left[\mathbf{S}^{2}\right]+\rho E[|\mathbf{S}|]$ is selected, namely, $\dot{\mathbf{V}} \leq 0$, according to Barbalat' Lemma [38], system (1) and system (3) can achieve synchronization under the controller law in (17).

This completes the proof.

\section{Simulation}

In this part, to confirm the validity of proposed method, we numerically examine the synchronization between fractional-order Chen system [39] and fractional-order Lü system [40]. In the simulation, step-by-step method is performed to receive numerical solution, and the detailed descriptions of this algorithm are available in [41, 42].

Here, we assume that the Chen system drives the Lü system. Hence, the driving system (fractional-order Lü system) is described as

$$
\begin{aligned}
& D^{\alpha} x_{1}=a_{1}\left(x_{2}-x_{1}\right)+h_{1}(\mathbf{x}, t) \dot{W}_{1}, \\
& D^{\alpha} x_{2}=-x_{1} x_{3}+c_{1} x_{2}+h_{2}(\mathbf{x}, t) \dot{W}_{2}, \\
& D^{\alpha} x_{3}=x_{1} x_{2}-b_{1} x_{3}+h_{3}(\mathbf{x}, t) \dot{W}_{3}
\end{aligned}
$$

which can also be written as

$$
D^{\alpha} \mathbf{x}=A_{1} \mathbf{x}+\mathbf{f}_{1}(\mathbf{x})+\mathbf{h}(t, \mathbf{x}) \dot{\mathbf{W}}
$$




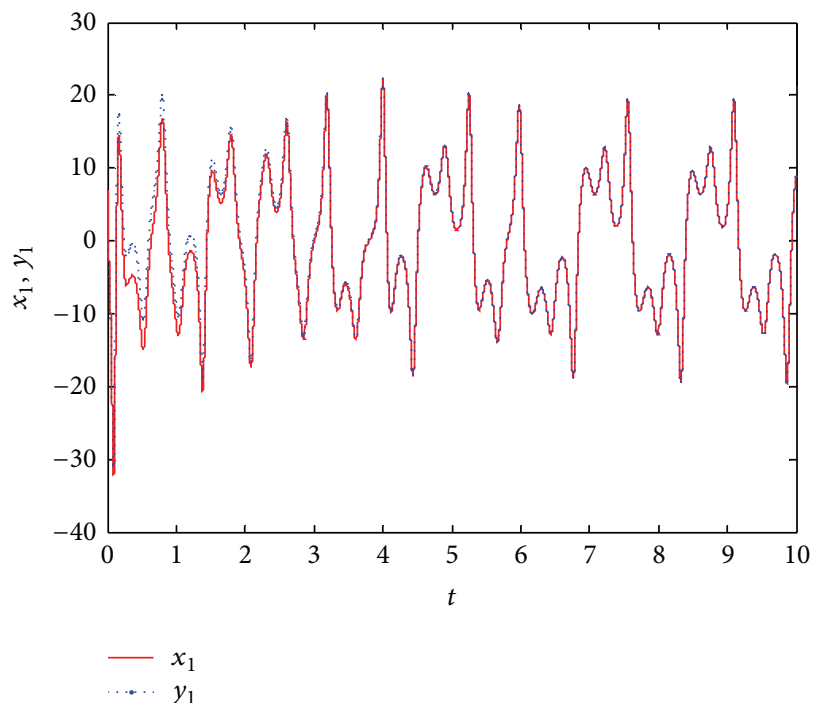

(a)

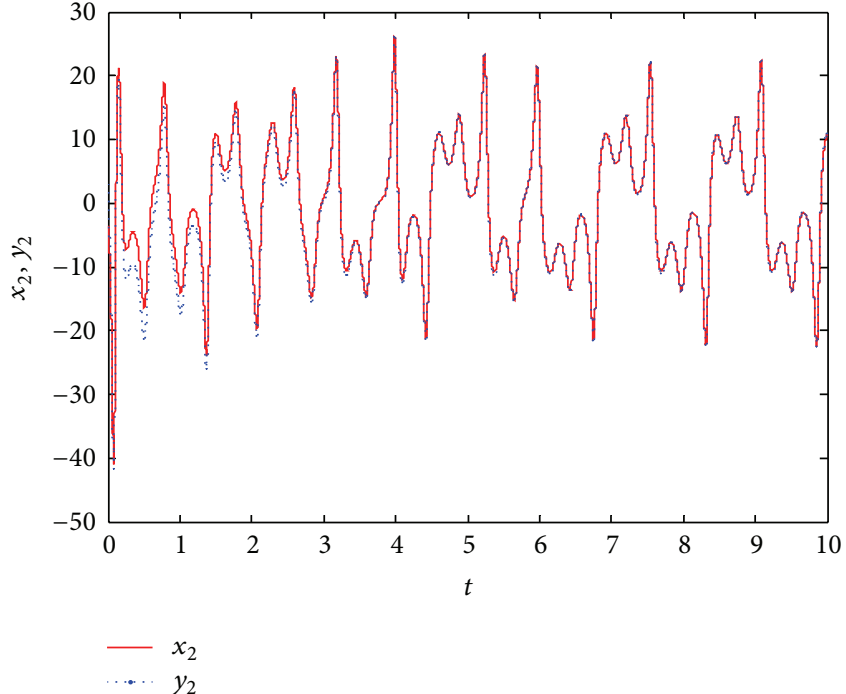

(b)

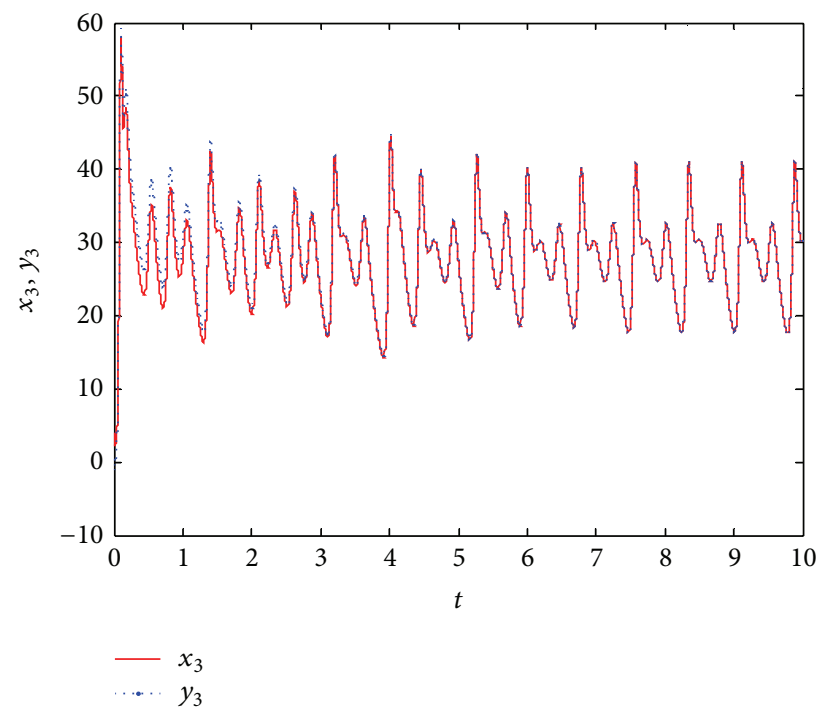

(c)

Figure 1: (a) The state trajectories of the system (26) and system (31) with the sliding mode control method. (Signals $\left.x_{1} ; y_{1}\right)$. (b) The state trajectories of the system (26) and system (31) with the sliding mode control method. (Signals $x_{2} ; y_{2}$ ). (c) The state trajectories of the system (26) and system (31) with the sliding mode control method. (Signals $x_{3} ; y_{3}$ ).

in which,

$$
\begin{gathered}
\mathbf{x}=\left(\begin{array}{c}
x_{1} \\
x_{2} \\
x_{3}
\end{array}\right), \quad A_{1}=\left[\begin{array}{ccc}
-a_{1} & a_{1} & 0 \\
0 & c_{1} & 0 \\
0 & 0 & -b_{1}
\end{array}\right], \\
\mathbf{f}_{1}(\mathbf{x})=\left(\begin{array}{c}
0 \\
-x_{1} x_{3} \\
x_{1} x_{2}
\end{array}\right), \quad \mathbf{h}(t, \mathbf{x})=\left(\begin{array}{l}
h_{1}(t, \mathbf{x}) \\
h_{2}(t, \mathbf{x}) \\
h_{3}(t, \mathbf{x})
\end{array}\right), \\
\dot{\mathbf{W}}=\left(\begin{array}{l}
\dot{W}_{1} \\
\dot{W}_{2} \\
\dot{W}_{3}
\end{array}\right) .
\end{gathered}
$$

It has been shown that the fractional-order Lü system can demonstrate chaotic behavior [24] when $a_{1}=35, b_{1}=3$, $c_{1}=28$, and $\alpha=0.9$.

The response system (fractional-order Chen system) is given as follows:

$$
\begin{gathered}
D^{\alpha} y_{1}=a_{2}\left(y_{2}-y_{1}\right), \\
D^{\alpha} y_{2}=\left(c_{2}-a_{2}\right) y_{1}-y_{1} y_{3}+c_{2} y_{2}, \\
D^{\alpha} y_{3}=y_{1} y_{2}-b_{2} y_{3},
\end{gathered}
$$

which can be written in the following form:

$$
D^{\alpha} \mathbf{y}=A_{2} \mathbf{y}+\mathbf{f}_{2}(\mathbf{y}),
$$




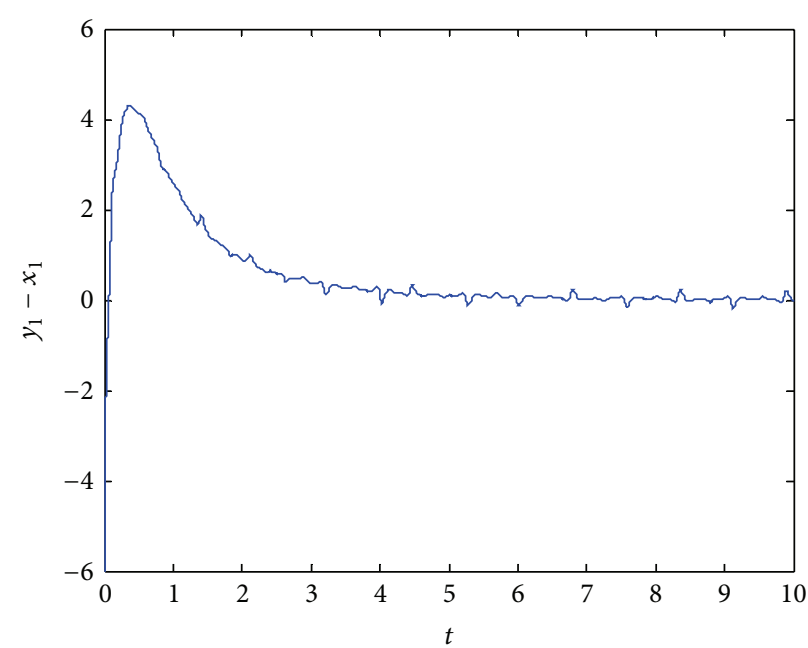

(a)

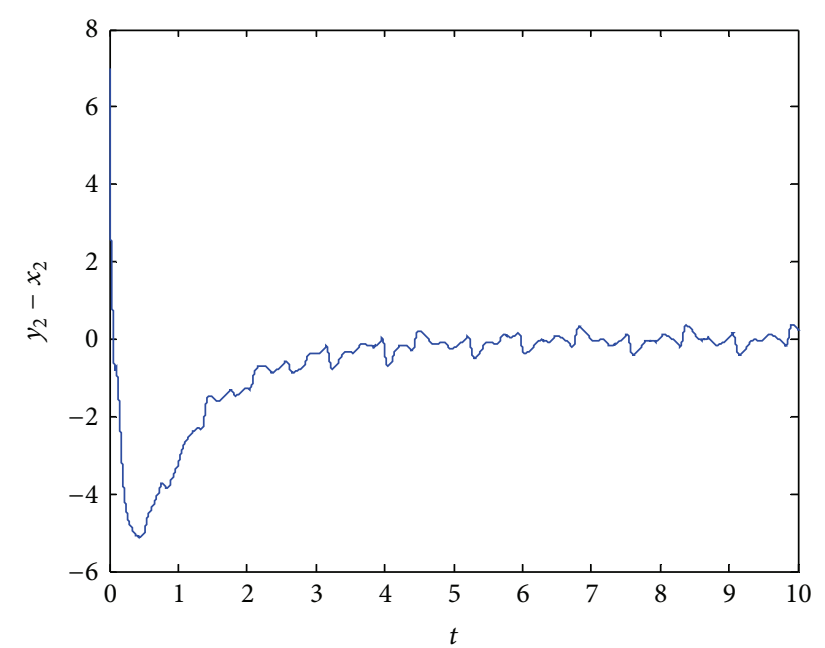

(b)

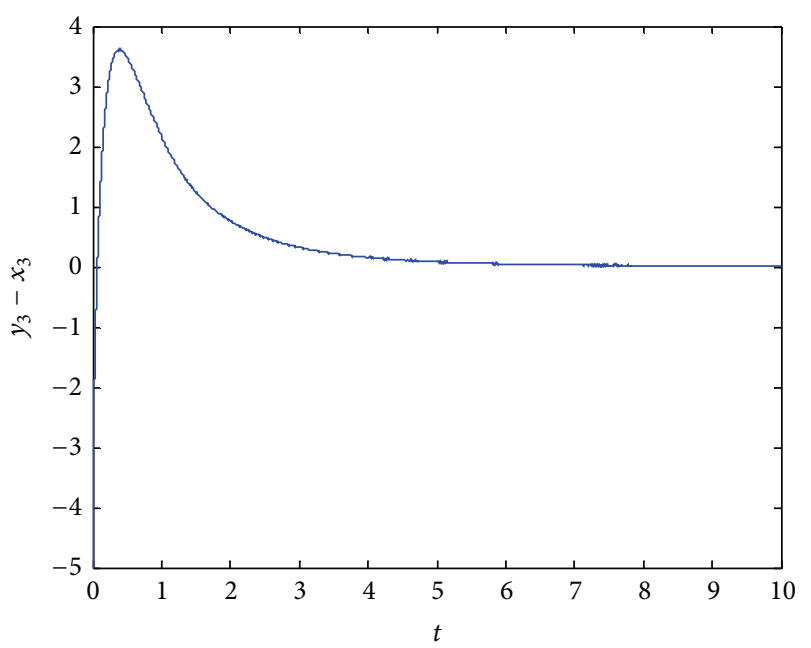

(c)

FIGURE 2: (a) The time evolution of synchronization error $e_{1}$ of the drive system (26) and response system (31). (b) The time evolution of synchronization error $e_{2}$ of the drive system (26) and response system (31). (c) The time evolution of synchronization error $e_{3}$ of the drive system (26) and response system (31).

where

$$
\begin{gathered}
\mathbf{y}=\left(\begin{array}{l}
y_{1} \\
y_{2} \\
y_{3}
\end{array}\right), \quad A_{2}=\left[\begin{array}{ccc}
-a_{2} & a_{2} & 0 \\
c_{2}-a_{2} & c_{2} & 0 \\
0 & 0 & -b_{2}
\end{array}\right], \\
\mathbf{f}_{2}(\mathbf{y})=\left(\begin{array}{c}
0 \\
-y_{1} y_{3} \\
y_{1} y_{2}
\end{array}\right),
\end{gathered}
$$

in which system will exhibit chaotic behavior [24] when $a_{2}=$ $35, b_{2}=3, c_{2}=28$, and $\alpha=0.9$. According to (3), the response system with controller can be described as follows:

$$
D^{\alpha} \mathbf{y}=A_{2} \mathbf{y}+\mathbf{f}_{2}(\mathbf{y})+\mathbf{u}(t)
$$

where $\mathbf{u}(t)=\left[u_{1}, u_{2}, u_{3}\right]^{T}$ is the control vector.

Now, we apply the proposed sliding control approach to finish synchronization between fractional-order Lü system driven by Gaussian white noise and fractional-order Chen system. Here, we define the error states as

$$
e_{i}=y_{i}-x_{i}
$$

and the sliding mode surface as

$$
\mathbf{S}=D^{\alpha-1} \mathbf{e}-\int_{0}^{t}\left(K+A_{2}\right) \mathbf{e}(\tau) d \tau,
$$

The control law is given by

$$
\mathbf{u}(t)=K \mathbf{e}-r \mathbf{S}-\rho \operatorname{sgn}(\mathbf{S})-\mathbf{f}_{2}(\mathbf{y})+\mathbf{f}_{1}(\mathbf{x})-\left(A_{2}-A_{1}\right) \mathbf{x} .
$$

In the numerical simulations, the initial conditions are set as $\left(x_{1}(0), x_{2}(0), x_{3}(0)\right)=(7,-4,4),\left(y_{1}(0), y_{2}(0), y_{3}(0)\right)=$ $(1,3,-1)$. The noise intensity matrices are presumably given in the form of $\left(h_{1}(t, \mathbf{x}), h_{2}(t, \mathbf{x}), h_{3}(t, \mathbf{x})\right)=(0.3,0.4, \sin t)$. 

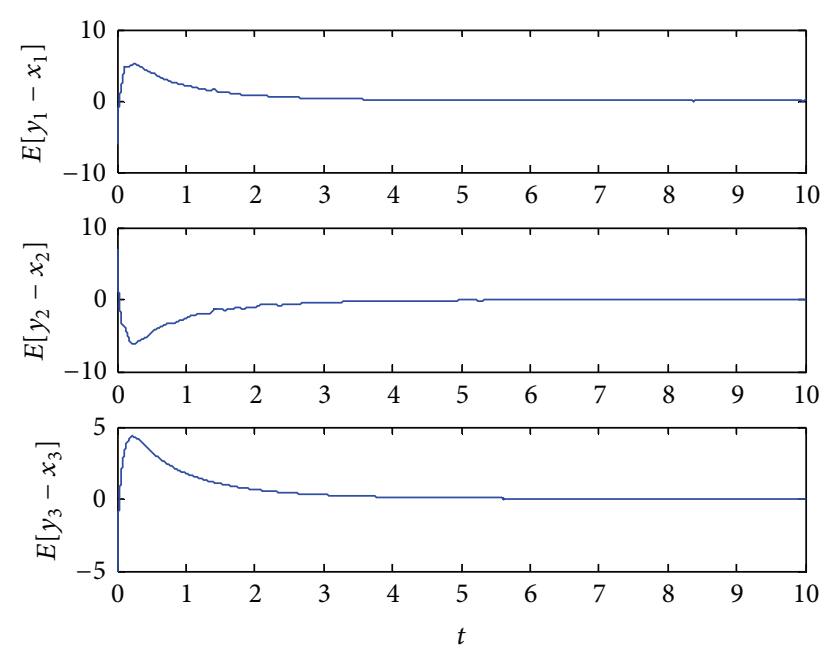

FIGURE 3: Mean value of synchronization errors $e_{1}, e_{2}, e_{3}$ between system (26) and system (31) using sliding mode method.
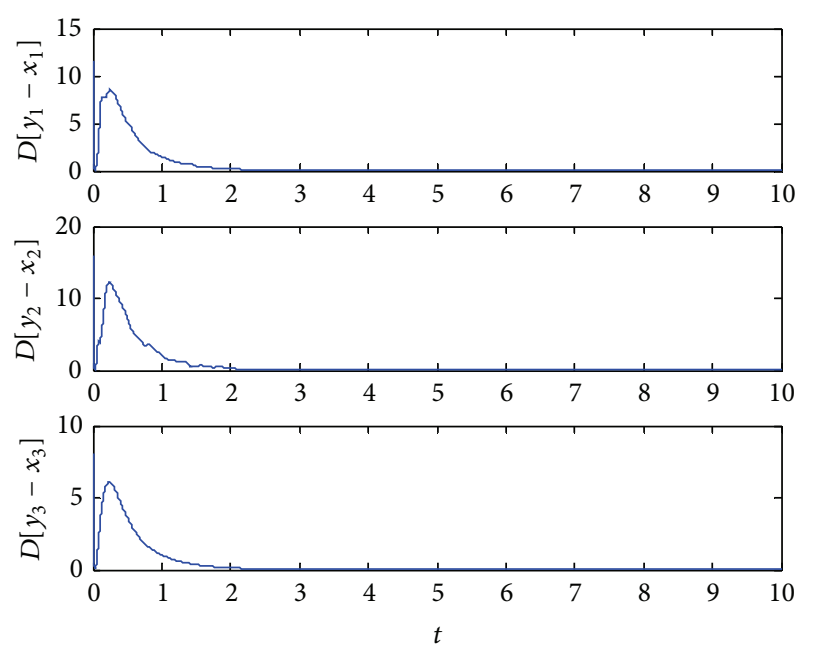

FIgURE 4: Variance of the state estimation errors $e_{1}, e_{2}, e_{3}$ of the system (26) and system (31) using the sliding mode method.

In fact, controller parameters can be chosen as $r=5, \rho=0.5$, and $K=\left[\begin{array}{rrr}34 & -35 & 0 \\ 7 & -29 & 0 \\ 0 & 0 & 2\end{array}\right]$. The time step size is $h=0.0005$. Then the simulation results are summarized in Figures 1-4. The state trajectories of the system (26) and system (31) under the sliding mode control method are shown in Figure 1(a) (signals $x_{1} ; y_{1}$ ), Figure 1(b) (signals $x_{2} ; y_{2}$ ), and Figure 1(c) (signals $x_{3} ; y_{3}$ ), respectively. Note that the driving system is shown by solid line whereas response system is shown by dashed line. As one can see, the designed controller is effectively capable achieving the synchronization of fractional-order Chen chaotic system; that is, the state variables $\left(y_{1}, y_{2}, y_{3}\right)$ follow the trail of $\left(x_{1}, x_{2}, x_{3}\right)$ well. Then the synchronization errors between the uncertain fractional-order chaotic Lü system and fractional-order chaotic Chen system are depicted in Figure 2(a) (signal $e_{1}$ ), and Figure 2(b) (signal $e_{2}$ ), Figure 2(c) (signal $e_{3}$ ). As it is expected, the synchronization errors (32) close to zero. Further, the expectation and variance of error vectors $e_{1}, e_{2}$ and $e_{3}$ converge to zero, as displayed in Figures 3 and 4 , which all indicate that the chaos synchronization between uncertain fractional-order chaotic Lü and Chen systems are indeed realized.

From the simulation results, it can be concluded that the obtained theoretic results are efficient and feasible for synchronizing fractional uncertain dynamical systems, and the proposed controller guarantees the convergence of the error system.

\section{Conclusions}

In this paper, we focus on the problem of synchronization between fractional-order chaotic systems with Gaussian fluctuation by the method of fractional-order sliding mode control. A fractional sliding surface is introduced, and the sliding mode controller is proposed for synchronization. Furthermore, convergence property has been analyzed for the error dynamics after adding proposed controllers. It has been shown that the fractional-order chaotic systems under uncertain environment can achieve synchronization by proper choice of control parameters $(r$ and $\rho$ ). Finally, to further illustrate the effectiveness of the proposed controllers, one applies the presented algorithm to the fractional-order Chen and fractional-order Lü systems through numerical simulations. From the simulation results, it is obvious that a satisfying control performance can be achieved by using the proposed method.

\section{Acknowledgments}

This work was supported by the NSF of China (Grant nos. 11372247 and 11102157), Program for NCET, the Shaanxi Project for Young New Star in Science \& Technology, NPU Foundation for Fundamental Research, and SRF for ROCS, SEM.

\section{References}

[1] R. Zhang and S. Yang, "Robust synchronization of two different fractional-order chaotic systems with unknown parameters using adaptive sliding mode approach," Nonlinear Dynamics, vol. 71, pp. 269-278, 2013.

[2] X. Wang, X. Zhang, and C. Ma, "Modified projective synchronization of fractional-order chaotic systems via active sliding mode control," Nonlinear Dynamics, vol. 69, pp. 511-517, 2012.

[3] P. Zhou, R. Ding, and Y. Cao, "Multi drive-one response synchronization for fractional-order chaotic systems," Nonlinear Dynamics, vol. 70, pp. 1263-1271, 2012.

[4] C. P. Li, W. H. Deng, and D. Xu, "Chaos synchronization of the Chua system with a fractional order," Physica A, vol. 360, no. 2, pp. 171-185, 2006.

[5] K. P. Wilkie, C. S. Drapaca, and S. Sivaloganathan, "A nonlinear viscoelastic fractional derivative model of infant hydrocephalus," Applied Mathematics and Computation, vol. 217, no. 21, pp. 8693-8704, 2011.

[6] Y. Luo, Y. Chen, and Y. Pi, "Experimental study of fractional order proportional derivative controller synthesis for fractional order systems," Mechatronics, vol. 21, no. 1, pp. 204-214, 2011. 
[7] M. Rivero, J. J. Trujillo, L. Vázquez, and M. Pilar Velasco, "Fractional dynamics of populations," Applied Mathematics and Computation, vol. 218, no. 3, pp. 1089-1095, 2011.

[8] N. Laskin, "Fractional market dynamics," Physica A, vol. 287, no. 3-4, pp. 482-492, 2000.

[9] W. M. Ahmad and J. C. Sprott, "Chaos in fractional-order autonomous nonlinear systems," Chaos, Solitons and Fractals, vol. 16, no. 2, pp. 339-351, 2003.

[10] T. T. Hartley, C. F. Lorenzo, and H. K. Qammer, "Chaos in a fractional order Chua's system," IEEE Transactions on Circuits and Systems I, vol. 42, no. 8, pp. 485-490, 1995.

[11] C. Li and G. Chen, "Chaos and hyperchaos in the fractionalorder Rössler equations,” Physica A, vol. 341, no. 1-4, pp. 55-61, 2004.

[12] C. Li and G. Peng, "Chaos in Chen's system with a fractional order," Chaos, Solitons and Fractals, vol. 22, no. 2, pp. 443-450, 2004.

[13] L.-J. Sheu, H.-K. Chen, J.-H. Chen et al., "Chaos in the NewtonLeipnik system with fractional order," Chaos, Solitons and Fractals, vol. 36, no. 1, pp. 98-103, 2008.

[14] P. Zhou and R. Ding, "Modified function projective synchronization between different dimension fractional-order chaotic systems," Abstract and Applied Analysis, vol. 2012, Article ID 862989, 12 pages, 2012.

[15] A. Kiani-B, K. Fallahi, N. Pariz, and H. Leung, "A chaotic secure communication scheme using fractional chaotic systems based on an extended fractional Kalman filter," Communications in Nonlinear Science and Numerical Simulation, vol. 14, no. 3, pp. 863-879, 2009.

[16] L. J. Sheu, "A speech encryption using fractional chaotic systems," Nonlinear Dynamics, vol. 65, no. 1-2, pp. 103-108, 2011.

[17] N. Singh and A. Sinha, "Optical image encryption using fractional Fourier transform and chaos," Optics and Lasers in Engineering, vol. 46, no. 2, pp. 117-123, 2008.

[18] N. Zhou, Y. Wang, L. Gong, H. He, and J. Wu, "Novel singlechannel color image encryption algorithm based on chaos and fractional Fourier transform," Optics Communications, vol. 284, no. 12, pp. 2789-2796, 2011.

[19] J. Lu, X. Wu, and J. Lü, "Synchronization of a unified chaotic system and the application in secure communication," Physics Letters A, vol. 305, no. 6, pp. 365-370, 2002.

[20] J. Lü and G. Chen, "A time-varying complex dynamical network model and its controlled synchronization criteria," IEEE Transactions on Automatic Control, vol. 50, no. 6, pp. 841-846, 2005.

[21] C. Li and W. Deng, "Chaos synchronization of fractional-order differential systems," International Journal of Modern Physics B, vol. 20, no. 7, pp. 791-803, 2006.

[22] S. Bhalekar and V. Daftardar-Gejji, "Synchronization of different fractional order chaotic systems using active control," Communications in Nonlinear Science and Numerical Simulation, vol. 15, no. 11, pp. 3536-3546, 2010.

[23] R. Zhang and S. Yang, "Adaptive synchronization of fractionalorder chaotic systems via a single driving variable," Nonlinear Dynamics, vol. 66, no. 4, pp. 831-837, 2011.

[24] Z. M. Odibat, "Adaptive feedback control and synchronization of non-identical chaotic fractional order systems," Nonlinear Dynamics, vol. 60, no. 4, pp. 479-487, 2010.

[25] D.-Y. Chen, Y.-X. Liu, X.-Y. Ma, and R.-F. Zhang, "Control of a class of fractional-order chaotic systems via sliding mode," Nonlinear Dynamics, vol. 67, no. 1, pp. 893-901, 2012.
[26] J. G. Lu, "Synchronization of a class of fractional-order chaotic systems via a scalar transmitted signal," Chaos, Solitons and Fractals, vol. 27, no. 2, pp. 519-525, 2006.

[27] I. Boiko, L. Fridman, R. Iriarte, A. Pisano, and E. Usai, "Parameter tuning of second-order sliding mode controllers for linear plants with dynamic actuators," Automatica, vol. 42, no. 5, pp. 833-839, 2006.

[28] J. J. Slotine and W. Li, Applied Nonlinear Control, Prentice Hall, Upper Saddle River, NJ, USA, 1991.

[29] M. S. Tavazoei and M. Haeri, "Synchronization of chaotic fractional-order systems via active sliding mode controller," Physica A, vol. 387, no. 1, pp. 57-70, 2008.

[30] M. Aghababa, "Finite-time chaos control and synchronization of fractional-order nonautonomous chaotic (hyperchaotic) systems using fractional nonsingular terminal sliding mode technique," Nonlinear Dynamics, vol. 69, pp. 247-261, 2012.

[31] A. S. Pikovsky, "Comment on 'chaos, noise, and synchronization,' Physical Review Letters, vol. 73, no. 21, p. 2931, 1994.

[32] K. H. Nagai and H. Kori, "Noise-induced synchronization of a large population of globally coupled nonidentical oscillators," Physical Review E, vol. 81, no. 6, Article ID 065202, 2010.

[33] H. Herzel and J. Freund, "Chaos, noise, and synchronization reconsidered," Physical Review E, vol. 52, no. 3, pp. 3238-3241, 1995.

[34] C.-H. Lai and C. Zhou, "Synchronization of chaotic maps by symmetric common noise," Europhysics Letters, vol. 43, no. 4, pp. 376-380, 1998.

[35] J.-S. Lin, J.-J. Yan, and T.-L. Liao, "Chaotic synchronization via adaptive sliding mode observers subject to input nonlinearity," Chaos, Solitons and Fractals, vol. 24, no. 1, pp. 371-381, 2005.

[36] H.-T. Yau, "Design of adaptive sliding mode controller for chaos synchronization with uncertainties," Chaos, Solitons and Fractals, vol. 22, no. 2, pp. 341-347, 2004.

[37] H. Salarieh and A. Alasty, "Chaos synchronization of nonlinear gyros in presence of stochastic excitation via sliding mode control," Journal of Sound and Vibration, vol. 313, no. 3-5, pp. 760-771, 2008.

[38] G. Tao, "A simple alternative to the Barbarlat lemma," IEEE Transactions on Automatic Control, vol. 42, no. 5, p. 698, 1997.

[39] J. Lü, G. Chen, D. Cheng, and S. Celikovsky, "Bridge the gap between the Lorenz system and the Chen system," International Journal of Bifurcation and Chaos in Applied Sciences and Engineering, vol. 12, no. 12, pp. 2917-2926, 2002.

[40] J. Lü and G. Chen, "A new chaotic attractor coined," International Journal of Bifurcation and Chaos in Applied Sciences and Engineering, vol. 12, no. 3, pp. 659-661, 2002.

[41] I. Petráš, Fractional-Order Nonlinear Systems Modeling, Analysis and Simulation, Springer, Berlin, Germany, 2011.

[42] T. Škovránek, I. Podlubny, and I. Petráš, "Modeling of the national economies in state-space: a fractional calculus approach," Economic Modelling, vol. 29, pp. 1322-1327, 2012. 


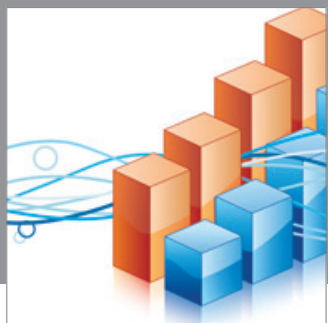

Advances in

Operations Research

mansans

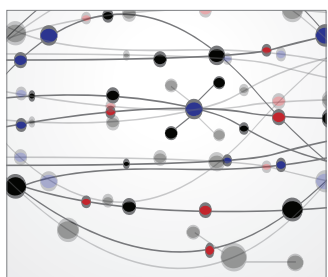

The Scientific World Journal
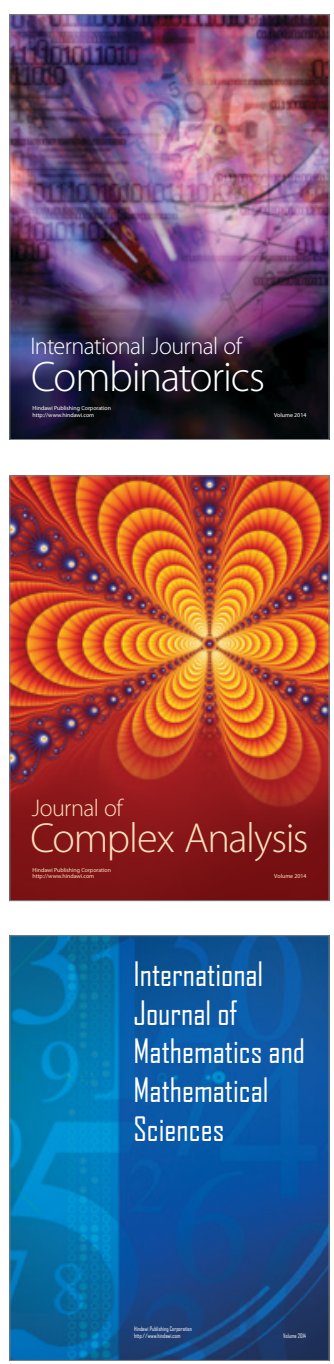
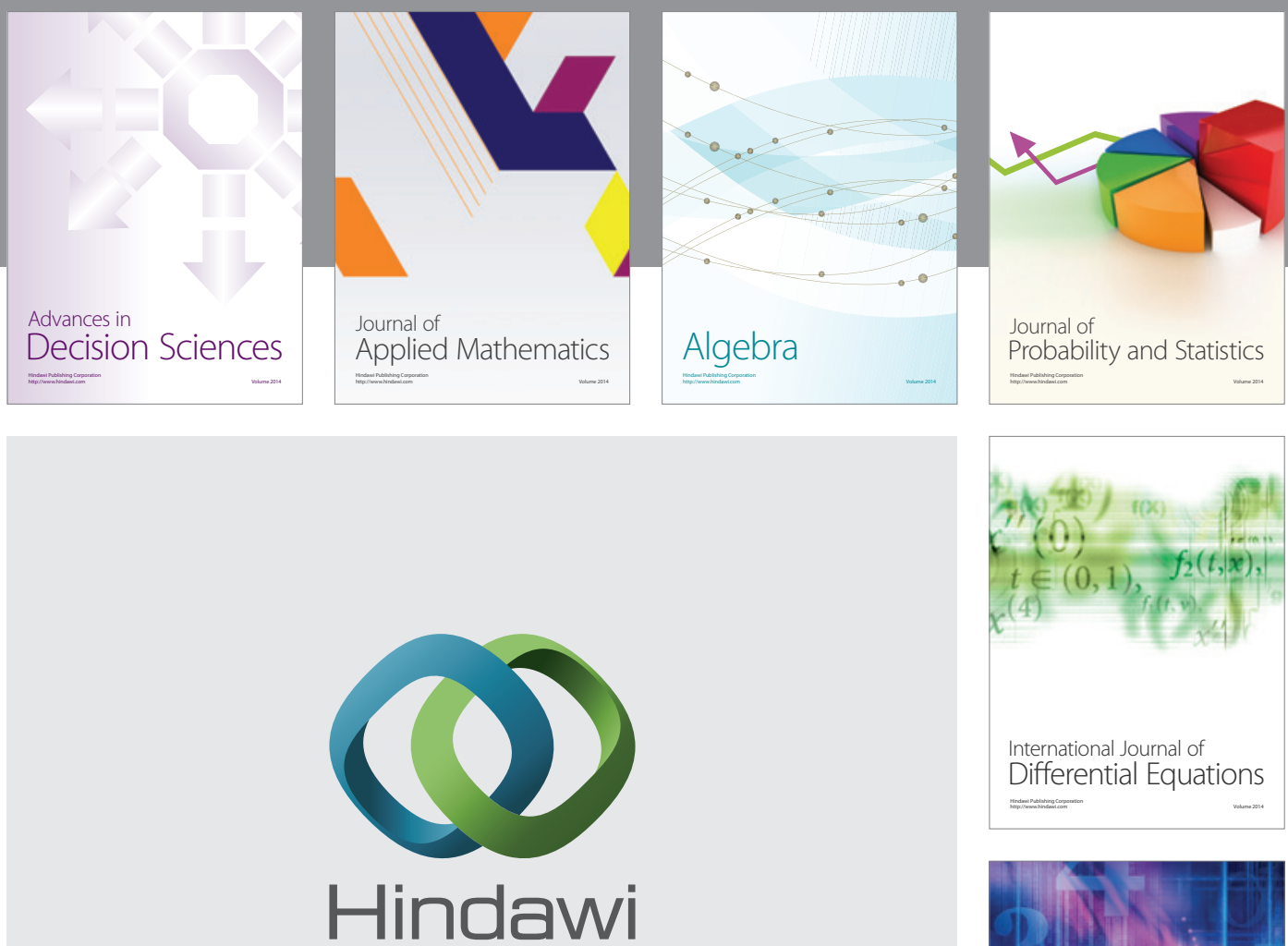

Submit your manuscripts at http://www.hindawi.com
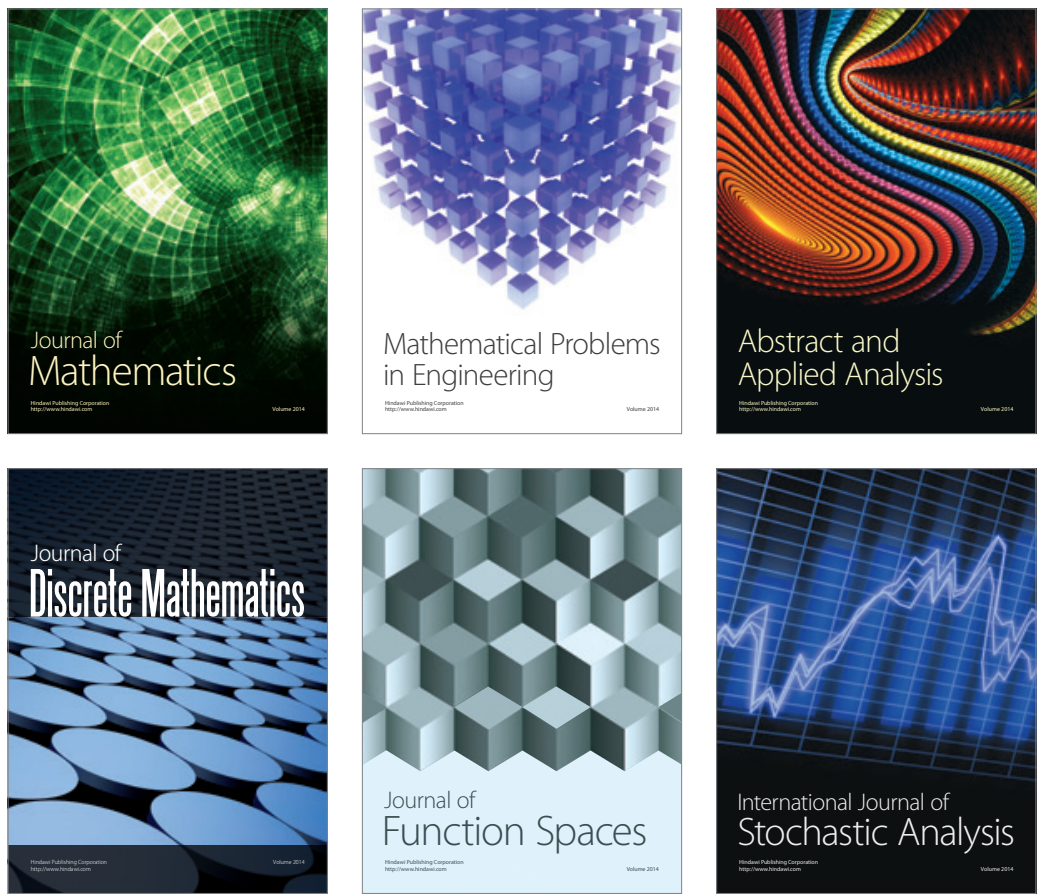

Journal of

Function Spaces

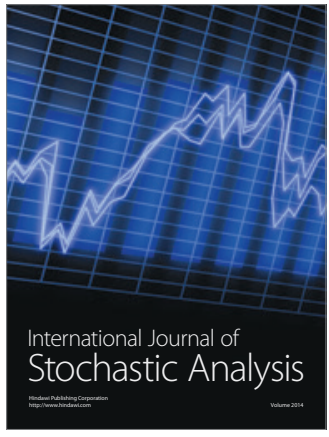

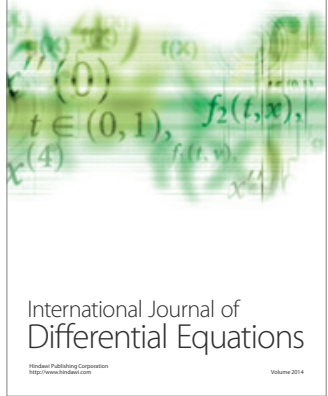
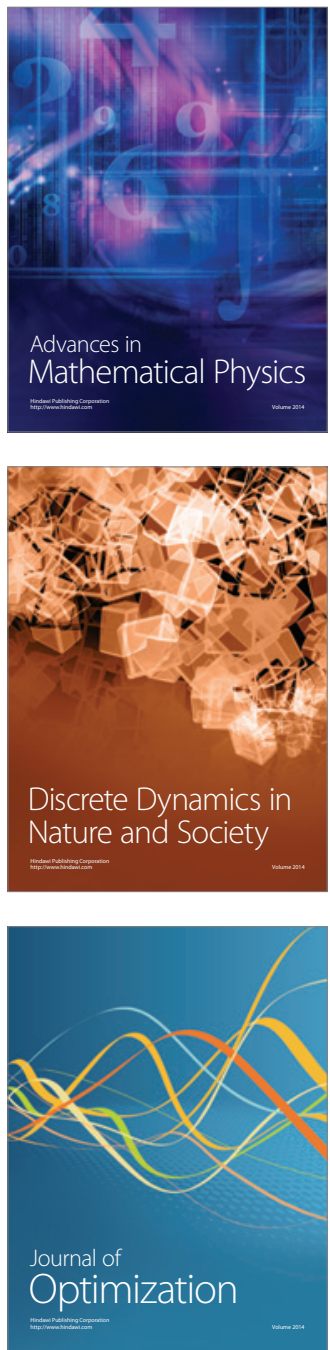